\title{
Enhanced fluoride removal by hydroxyapatite-modified activated alumina
}

\author{
G. Tomar $\cdot$ A. Thareja $\cdot$ S. Sarkar
}

Received: 25 January 2014/Revised: 7 May 2014/ Accepted: 12 July 2014/Published online: 25 July 2014

(C) Islamic Azad University (IAU) 2014

\begin{abstract}
Fluoride in drinking water is beneficial at low concentrations but is considered harmful for the human health when present at concentrations exceeding $1.5 \mathrm{mg} / \mathrm{L}$. Prevalence of high concentration of fluoride in drinking water, combined with the nonavailability of alternate viable sources, makes the treatment of the contaminated water an essential task. In this study, we report the synthesis and characterization of a hybrid adsorbent; hydroxyapatitemodified activated alumina (HMAA) prepared by dispersing nanoparticles of hydroxyapatite inside activated alumina granules. The composite adsorbent provided a synergy toward fluoride removal from contaminated drinking water. The hybrid adsorbent possesses a maximum adsorption capacity of $14.4 \mathrm{mg} \mathrm{F} / \mathrm{g}$ which is at least five times higher than the virgin-activated alumina, which has been used extensively for fluoride removal. HMAA was regenerated using six bed volumes of a solution containing commonly available innocuous chemicals. The adsorbent was subjected to multiple numbers of operating cycles within a column, each cycle consisting of one adsorption run followed by regeneration.
\end{abstract}

Electronic supplementary material The online version of this article (doi:10.1007/s13762-014-0653-5) contains supplementary material, which is available to authorized users.

\section{G. Tomar}

Department of Biotechnology and Environmental Sciences,

Thapar University, Patiala 147004, India

A. Thareja - S. Sarkar $(\bowtie)$

Department of Civil Engineering, Indian Institute of Technology

Roorkee, Roorkee 247667, India

e-mail: srkarfce@iitr.ac.in
Keywords Activated alumina $\cdot$ Hydroxyapatite-modified activated alumina $\cdot$ Fluoride, defluoridation .

Nanoparticles, adsorption $\cdot$ Column run $\cdot$ Regeneration

\section{Introduction}

Groundwater remains the only choice in many developed as well as developing countries such as India for fulfilling the daily needs and demands of people. But unfortunately, due to weathering of rocks and over-exploitation, there is leaching of minerals in the groundwater that usually results in the release of extra dissolved species into the groundwater. Arsenic and fluoride are known to be major geogenic pollutants that cause adverse effects on human health (Farooqi et al. 2007). Although fluoride contamination in the drinking water is not as fatal as arsenic; however, the widespread nature and debilitating effect on the human beings have made the fluoride contamination problem as the one which needs to be urgently remediated. One-fourth countries of the world and a large fraction (28\%) of rural population of the developing countries (India, Pakistan, Bangladesh, etc.) are facing the problems of water supply with unsafe level of fluoride, resulting in a condition which demands an immediate and robust solution. High fluoride concentrations in groundwater, up to more than $30 \mathrm{mg} / \mathrm{L}$, have been observed widely in many parts of the world (Azbar and Turkman 2000; Wang et al. 2002; Chernet et al. 2002). There are many districts in Indian states where the community is compelled to use water having concentration of fluoride as high as 5 to $49 \mathrm{mg} / \mathrm{L}$ while the permissible limit for fluoride intake decided by WHO is only $1.5 \mathrm{mg} / \mathrm{L}$ (World Health Organization 2008). In cases where there is no viable alternative source for safe 
drinking water, it is essential to have a treatment system in place before the water is used for drinking and cooking.

Various efforts have been undertaken to develop an effective method for fluoride removal from drinking water. The methods that are either developed in the laboratory or used in the field are based on one or combination of the following principles: adsorption (Apparao et al. 1998), ion exchange (Singh et al. 1999), precipitation-coagulation (Saha 1993; Rao et al. 1998; Reardon and Wang 2000) and membrane separation process (Mohapatra et al. 2009; Ndiaye et al. 2005; Richards et al. 2010; Malaisamy et al. 2011; Hu and Dickson 2006; Hou et al. 2010; Durmaz et al. 2005; Hichour et al. 2000; Kabay et al. 2008; Sahli et al. 2007, Meenakshi and Maheshwari 2006). A major point to be noted regarding the fluoride removal from contaminated water is that while other commonly occurring toxic ions in drinking water are significant in sub-mg/L concentration, fluoride normally is present in $\mathrm{mg} / \mathrm{L}$ range in the contaminated waters. Therefore, simply considering the stoichiometry, it is much more difficult to develop a cost-effective removal process for fluoride than other trace contaminants such as arsenic.

Adsorption using a plug-flow device such as an adsorption column is always user-friendly and desirable due to its inherent design supremacy compared to the completely mixed reactors generally used in precipitation-type fluoride removal. Due to this reason, adsorption using columns is a popular process for removal of contaminants from contaminated drinking water. Activated alumina (AA) is frequently used as an adsorbent for such plug-flow-type adsorption units employed for fluoride removal. The advantage of AA is that it is a regenerable media but it has low capacity for fluoride removal. Considering the fact that fluoride occurs in $\mathrm{mg} / \mathrm{L}$ concentration range, often the regeneration has to be performed rather frequently, generally after every 100-150 bed volumes of operation of the adsorption column. Although the regeneration can be done using commonly available chemicals, the frequent regeneration requirement tends to make the process cost-prohibitive. Therefore, there is natural demand for the development of adsorbent media with comparable cost but with a much superior adsorption capacity, so that an affordable and workable solution to the fluoride problem can be reached.

The other adsorbents previously studied for fluoride removal include bone char, activated carbon, activated bauxite, ion-exchange resins, fly ash, super phosphate and tricalcium phosphate, clays and soils, synthetic zeolites and other minerals (Ayoob et al. 2008). It was found that hydroxyapatite synthesized using locally available materials can effectively remove fluoride at considerable low costs. It is recognized that hydroxyapatite holds potential as a possible solution to the fluoride problem (Sundaram et al. 2009). Hydroxyapatite substrate can also be made from bone char, but consuming water filtered with animal bones conflicts with religious and culture taboos in many places in the world, especially in India. On the other hand, synthetic nano-hydroxyapatite $\left[\mathrm{Ca}_{5}\left(\mathrm{PO}_{4}\right)_{3} \mathrm{OH}\right]$ particles showed a very good capacity for fluoride removal from contaminated waters (Gao et al. 2009; MacDonald et al. 2011). However, the nanoparticles, due to their poor durability and excessive pressure drop across the column, are not suitable for direct application in fixed bed columns, reactive barriers, or any plug-flow-like applications (Sarkar et al. 2012).

Furthermore, in the nanoscale, the particles typically tend to aggregate, a property that negates many of the benefits associated with the nanoscale dimensions. One way to make nanoparticles fit for the field applications is to encapsulate them inside a matrix of a host material (Sarkar et al. 2012). As a host material, AA is a good choice for encapsulation of hydroxyapatite. Porous AA granules not only shall impart excellent mechanical strength, durability and hydraulic properties to the composite adsorbent; being an adsorbent itself, it also should add synergy to the overall adsorption capacity of the composite adsorbent. The composite material should be regenerable, so that suitability for regeneration and reuse of the adsorbent over multiple numbers of cycles shall bring cost-effectiveness. Therefore, it is worth developing a hybrid adsorbent which shall consist of two phases-nanoparticles of hydroxyapatite and activated alumina. In this article, we report the synthesis and characterization of a hybrid adsorbent, hydroxyapatite-modified activated alumina (HMAA), prepared by dispersing nanoparticles of hydroxyapatite inside AA granules. We also report the results of performance validation experiments for this hybrid adsorbent, HMAA, for fluoride removal from synthetic contaminated water under different operating conditions. We also developed and validated suitable regeneration protocols for exhausted hybrid adsorbent. We have also reported the results of column runs with the adsorbent over multiple number of cycles.

\section{Materials and methods}

Reagents and chemicals

Activated alumina (AA) was obtained from Oxide India Private Ltd, Durgapur, India. The AA used in the study was 
spherical with sizes between 600 and 900 micron, with available surface area of $250 \mathrm{~m}^{2} / \mathrm{g}$. The chemicals required for aqueous phase determination of fluoride, such as SPADNS reagent and zirconyl oxychloride octahydrate, were procured from LOBA Chemicals, India; sodium fluoride, sodium chloride and sodium sulfate from QUALIGENS, India; sodium hydroxide pellets, ammonium dihydrogen phosphate and calcium chloride from HIMEDIA, India. All the chemicals used were of analytical reagent grade.

Synthesis of hydroxyapatite-modified activated alumina (HMAA)

Figure 1 schematically shows the steps involved in the synthesis of HMAA from commonly available chemicals. Briefly, $5 \mathrm{~g}$ of virgin AA granules was carefully washed in deionized water several times and dried in an oven at $105{ }^{\circ} \mathrm{C}$ for $4 \mathrm{~h}$. Next, $500 \mathrm{~mL}$ of $5 \%$ solution of ammonium dihydrogen phosphate with $\mathrm{pH}$ adjusted to four was circulated for about 15 min through a glass column loaded with precleaned AA granules. Subsequently, the solution is decanted, and $1 \mathrm{~L}$ of $5 \mathrm{~N}$ solution of calcium chloride with $\mathrm{pH}$ adjusted to 11 was circulated through the column for another $15 \mathrm{~min}$. After this step, the contents of the column were thoroughly washed with deionized water flowing through the column for about $10 \mathrm{~min}$. The whole procedure was repeated for two cycles.

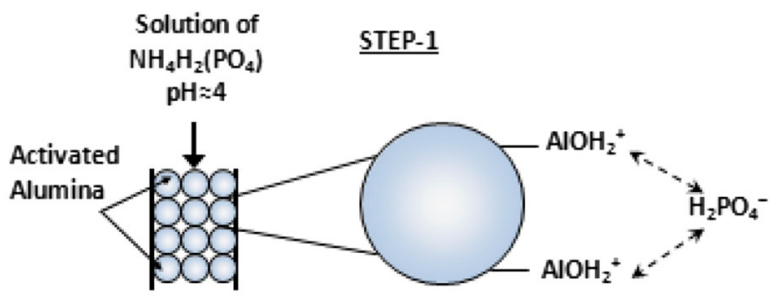

STEP-2

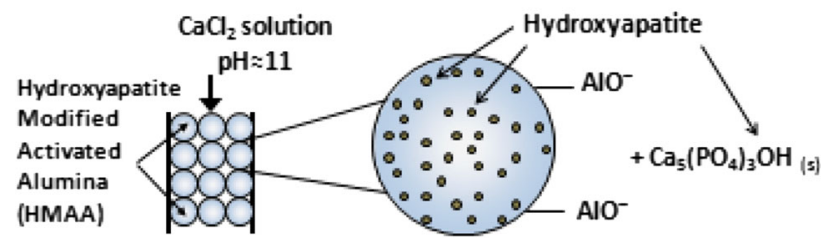

Fig. 1 Synthesis of hydroxyapatite-modified activated alumina by dispersing particles of hydroxyapatite inside the pores of activated alumina granules
Characterization of the hydroxyapatite-modified activated alumina (HMAA)

The HMAA prepared in the foregoing step was characterized using a scanning electron microscope (SEM), Quanta 200 F (FEI, USA), equipped with capability of performing energy-dispersive $\mathrm{x}$-ray (EDX) of the samples. The synthesized HMAA was also analyzed for Fourier transform infrared spectroscopy (FTIR) using NICOLET 6700 (Thermo Scientific, USA). FTIR spectra of the solid samples were performed by diluting them in $\mathrm{KBr}$ pellets. The results of the FTIR spectroscopy were used to determine the nature of functional groups present in HMAA.

Fluoride sorption-desorption studies

The fluoride sorption and desorption experiments were carried out using the HMAA granules synthesized in the laboratory. The experiments were carried out in both batches as well as column mode using synthetic solution containing fluoride in the background of other competing ions. For all the studies, the background of commonly occurring competing anions comprised of chloride, sulfate and bicarbonate ions maintained at concentrations much higher than that of fluoride, so that the synthetic solution resembles a realistic contaminated ground water sample. All batch adsorption experiments were carried out using well-stirred solutions containing fluoride initially at concentration of $10 \mathrm{mg} / \mathrm{L}$. For isotherm studies, $200 \mathrm{~mL}$ of the solution at near-neutral $\mathrm{pH}$ was placed in small plastic bottles with varied amount of HMAA and was kept on a platform shaker for $8 \mathrm{~h}$. A kinetic study indicated that the equilibrium is reached within $8 \mathrm{~h}$ of contact time. After $8 \mathrm{~h}$ of shaking, the liquid samples were taken out and filtered through glass fiber filter and the filtrate was analyzed for fluoride content. Interferences of $\mathrm{pH}$ on the adsorption were studied at equilibrium by varying the initial $\mathrm{pH}$ of the solution either by adding $\mathrm{HCl}$ or $\mathrm{NaOH}$. Column studies were carried out using a bed of HMAA inside a 11-mmdiameter glass column, which was subjected to a continuous flow of a synthetic solution containing fluoride with background concentration of common anions. Desorption studies within the column for the fluoride-loaded adsorbents were carried out using $\mathrm{NaOH}$ solution at alkaline $\mathrm{pH}$.

Analysis of water samples

Fluoride content in the water samples was analyzed using UV-VIS spectrophotometer (Hitachi, U-2900) following the SPADNS method described in the Standard Methods 
(American Public Health Association 2012). Briefly, fluoride standards, samples or their aliquots were first diluted to $50 \mathrm{~mL}$ with distilled water followed by addition of $10 \mathrm{~mL}$ mixed acid-zirconyl-SPADNS reagent, which when mixed thoroughly produced characteristic red color. The concentration of fluoride in the sample was determined by analyzing their absorbance recorded at $570 \mathrm{~nm}$ using UV-VIS spectrophotometer.

\section{Results and discussion}

\section{Characterization of HMAA}

\section{Morphological studies using scanning electron micrograph} and energy-dispersive $x$-ray

Figure 2 shows a scanning electron micrograph (SEM) of the interior of HMAA synthesized in the laboratory. The heterogeneity in the surface inside the hybrid adsorbent clearly points out to the presence of two different phases inside the adsorbent; the small amorphous precipitates of hydroxyapatite of size predominantly less than $100 \mathrm{~nm}$ dispersed in the foreground of the matrix of AA particles of larger size. Figure 3 shows the results of EDX analysis of HMAA shown in Fig. 2. The significant relative abundance of calcium and phosphorus along with aluminum and oxygen in HMAA provides the evidence of presence of hydroxyapatite-like phases dispersed within the matrix of activated alumina, the parent adsorbent. A quantitative analysis of the EDX data showed that calcium-to-phosphorus ratio in the synthesized adsorbent was 1.42 , which is close to that present in calcium hydroxyapatite molecule having a formula of $\mathrm{Ca}_{5}\left(\mathrm{PO}_{4}\right)_{3} \mathrm{OH}$.

\section{Fourier transform infrared spectroscopy}

In order to further investigate the characteristics of the hybrid adsorbent, HMAA was subjected to Fourier transform infrared (FTIR) spectroscopy. FTIR spectra are a useful tool to help identify functional groups in a molecule as each specific chemical bond has a unique energy absorption band from which one can obtain structural and bond information on a complex in order to study the strength and fraction of hydrogen bonding and miscibility. Fig. A of electronic supplementary material shows the FTIR spectra of HMAA. The major bands for the HMAA can be assigned as follows: $3454 \mathrm{~cm}^{-1}$ (-OH stretching vibrations); 3,094 and $2098 \mathrm{~cm}^{-1}$ (P-OH stretching vibration $) ; 1747$ and $1638 \mathrm{~cm}^{-1}(\mathrm{O}=\mathrm{P}-\mathrm{OH}$ stretching vibration); 1375, 1232 and $1071 \mathrm{~cm}^{-1}(\mathrm{P}=\mathrm{O}$ stretching vibration); $743 \mathrm{~cm}^{-1}$ (P-O stretching vibration); and $612 \mathrm{~cm}^{-1}$ (OH-P-OH stretching vibration) (Stuart 2004; Rehman and Bonfield 1997).

The EDX and FTIR studies point out to the presence of hydroxyapatite phase inside the HMAA, whereas the SEM indicates that the hydroxyapatite phases are nanoparticles having dimensions less than $100 \mathrm{~nm}$. Therefore,

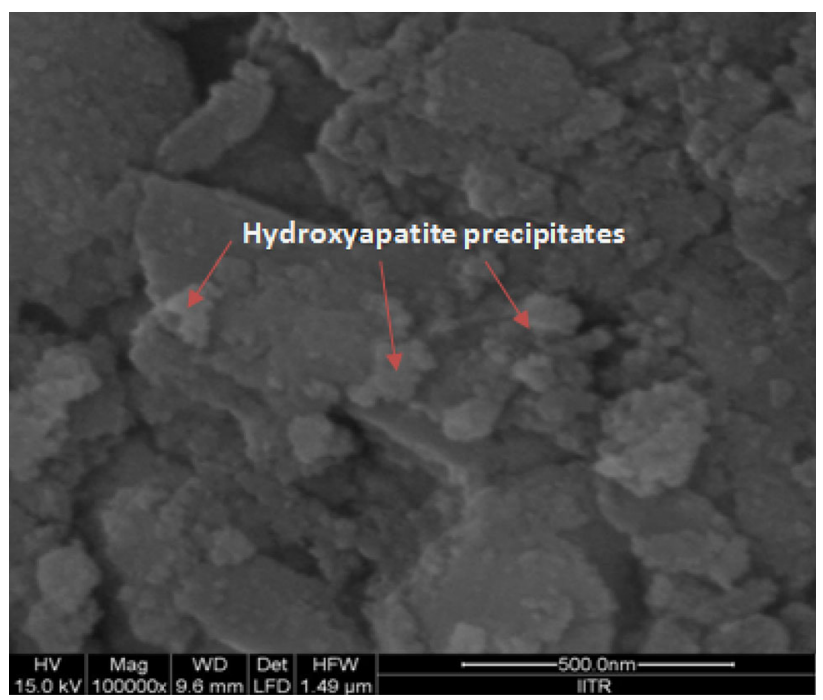

Fig. 2 Scanning electron photomicrograph magnification of hydroxyapatite-modified activated alumina at 50,000X

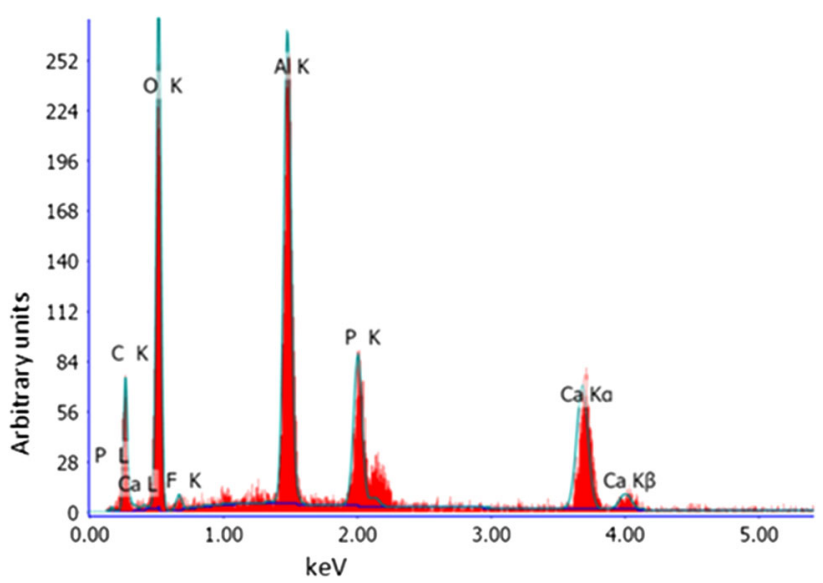

Fig. 3 Energy-dispersive X-ray analysis of surface of hydroxyapatite-modified activated alumina 
characterization studies conclusively prove the presence of nanoparticles of hydroxyapatite within the activated alumina matrix of HMAA synthesized in the laboratory.

Batch adsorption studies

Batch isotherm studies were carried out to determine the equilibrium adsorption capacities of the virgin AA and HMAA. A preliminary kinetic study showed that after around $8 \mathrm{~h}$ of contact time, equilibrium state is attained. So, for all isotherm studies, equilibration contact time of $8 \mathrm{~h}$ was used. Figure 4 shows comparative values of the equilibrium fluoride adsorption capacities of virgin AA and HMAA observed under identical experimental conditions. The synthetic fluoride-containing solution had background concentration of electrolytes containing commonly occurring anions such as chloride, sulfate and bicarbonate.

Following the batch experiments, the data were analyzed for the goodness of fit with two equilibrium adsorption models, namely Freundlich's model and Langmuir's model (Sundaram et al. 2008). The Freundlich model indicates the surface heterogeneity of the adsorbent and is defined as per the Eq. (1):

$q_{e}=k_{f} C_{e}^{\frac{1}{n}}$

where $k_{f}$ and $1 / n$ are Freundlich constants related to the adsorption capacity and adsorption intensity, respectively. $Q_{e}$ and $C_{e}$ are the equilibrium concentration of the adsorbate in adsorbent and aqueous phase, respectively.

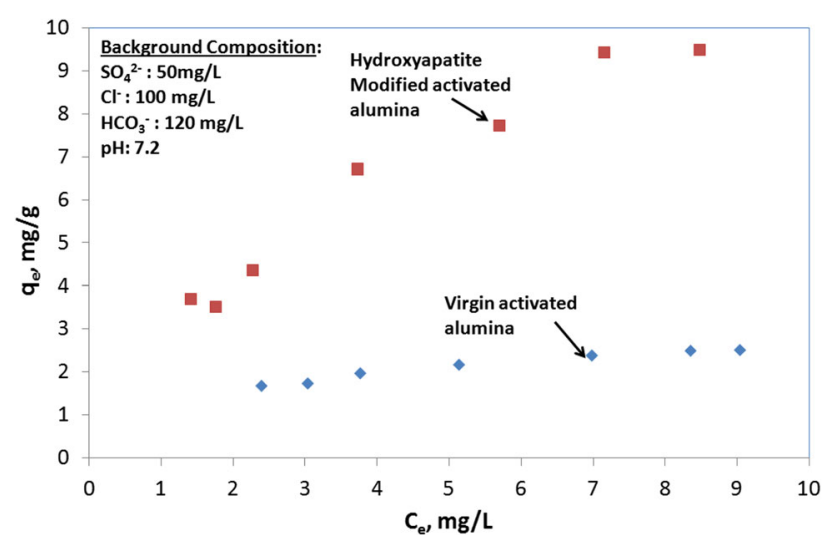

Fig. 4 Comparative equilibrium fluoride adsorption capacities of virgin and hydroxyapatite-modified activated alumina at different equilibrium fluoride concentrations with background electrolyte concentration
Taking natural logarithm on both the sides, the Eq. (1) transforms to the linear form as indicated in Eq. (2):

$\ln q_{e}=\ln k_{f}+\frac{1}{n} \ln C_{e}$

if the experimental data show a good correlation with the Eq. (2), then the adsorption process is said to follow the Freundlich model.

The Langmuir model is represented by the Eq. (3):

$q_{e}=\frac{Q_{\max } b C_{e}}{1+b C_{e}}$

where $Q_{\max }$ is the maximum amount of the fluoride uptake per unit weight of adsorbent to form a complete monolayer on the surface at a very high $C_{e}$, and $b$ is a constant related to the affinity of the binding sites. $Q_{\max }$ represents a particle limiting adsorption capacity when the surface is fully covered with adsorbate ions and assists in the comparison of adsorption performance, particularly in case where the sorbent did not reach its full saturation in experiments. A reorganization of the terms yields the Eq. (4) as follows:

$\frac{1}{q_{e}}=\left(\frac{1}{Q_{\max } b}\right)\left(\frac{1}{C_{e}}\right)+\left(\frac{1}{Q_{\max }}\right)$

The linear plot of $1 / C_{e}$ versus $1 / q_{e}$ for the experimental data indicates the applicability of Langmuir adsorption isotherm for the adsorption process. Table 1 shows the general equations of the two models and correlation of the observed data with the above two models, $R^{2}$ being the correlation coefficient showing the goodness of the

Table 1 Isotherms, goodness of fit for experimental data and values of constants and coefficients obtained from fluoride adsorption equilibrium studies performed with HMAA and AA

\begin{tabular}{lll}
\hline Isotherm & $\begin{array}{l}\text { Correlation coefficient } \\
\left(R^{2}\right)\end{array}$ & $\begin{array}{l}\text { Value of coefficients and } \\
\text { constants }\end{array}$ \\
\hline Langmuir & AA: 0.98 & $\begin{array}{l}Q_{\max }=3.1 \mathrm{mg} / \mathrm{g} \\
b=0.47 \mathrm{~L} / \mathrm{mg}\end{array}$ \\
& & $\begin{array}{l}Q_{\max }=14.4 \mathrm{mg} / \mathrm{g} \\
b=0.21 \mathrm{~L} / \mathrm{mg}\end{array}$ \\
& HMAA: 0.94 & $K_{f}=1.26$ \\
Freundlich & AA: 0.98 & $n=3.1$ \\
& & $K_{f}=2.79$ \\
& HMAA: 0.98 & $n=1.67$
\end{tabular}

$C_{e}$ is equilibrium fluoride concentration in aqueous phase, $\mathrm{mg} / \mathrm{L}$, and $q_{e}$ is equilibrium fluoride concentration in adsorbent phase, $\mathrm{mg} / \mathrm{g} ; A A$ activated alumina, $H M A A$ hydroxyapatite-modified activated alumina 
fit. Values of Langmuir constants $k_{f}$ and $n$ for virgin AA were 1.26 and 3.1, respectively. Values of Langmuir constants $Q_{\max }$ and $b$ were $3.1 \mathrm{mg} / \mathrm{g}$ and 0.47 $\mathrm{L} / \mathrm{mg}$, respectively. Langmuir model points out to a maximum achievable fluoride adsorption capacity of $3.1 \mathrm{mg} / \mathrm{g}$ by virgin AA. The values of the constants are consistent to those found by other researchers (Ghorai and Pant 2005). For HMAA, the values of Freundlich's adsorption model constants $k_{f}$ and $n$ were observed to be 2.79 and 1.67, respectively. Since the value of $n$ is greater than unity, the adsorption is favorable. Langmuir constants $Q_{\max }$ and $b$ observed were $14.4 \mathrm{mg} / \mathrm{g}$ and $0.21 \mathrm{~L} / \mathrm{mg}$, respectively, indicating a maximum adsorption capacity of $14.4 \mathrm{mg} / \mathrm{g}$ for the adsorbent even when there was competition from the other commonly occurring anions. From Table 1, it is clear that the adsorption behavior of both virgin AA and HMAA corresponded well with Langmuir and Freundlich model equations, which is a confirmation of surface heterogeneity as well as validation for monolayer sorption onto surface of both the adsorbents.

Comparison of the capacities of the adsorbents indicates that the fluoride adsorption capacity in the HMAA has increased by almost fivefold compared with virgin AA as a result of dispersal of hydroxyapatite within its pores. The boost to the adsorption capacity in HMAA compared with virgin AA is due to the dispersed phase of hydroxyapatite nanoparticles, which provided significant synergy to the adsorption capacity due to its high surface area to volume ratio.

\section{Effect of $\mathrm{pH}$ on adsorption of fluoride}

The $\mathrm{pH}$ of aqueous solution plays an important role in controlling the adsorption behavior of various inorganic adsorbents at the water-adsorbent interface. In order to study the effect of $\mathrm{pH}$ on the adsorption, batch adsorption experiments were carried out with solution at different $\mathrm{pH}$ containing fluoride concentration of $9.0 \mathrm{mg} / \mathrm{L}$ with background concentration of commonly occurring anions. The solutions were adjusted to different $\mathrm{pH}$ in the range of 4-10. The adsorbent dose was kept at $7 \mathrm{~g} / \mathrm{L}$. After reaching the equilibrium, final $\mathrm{pH}$ of the solutions were measured. Figure 5 shows the percentage adsorption of fluoride from the solution at different equilibrium $\mathrm{pH}$. The adsorption capacity was found to be maximum around $\mathrm{pH}$ of 8 . (Ghorai and Pant 2005) indicated that AA also follows

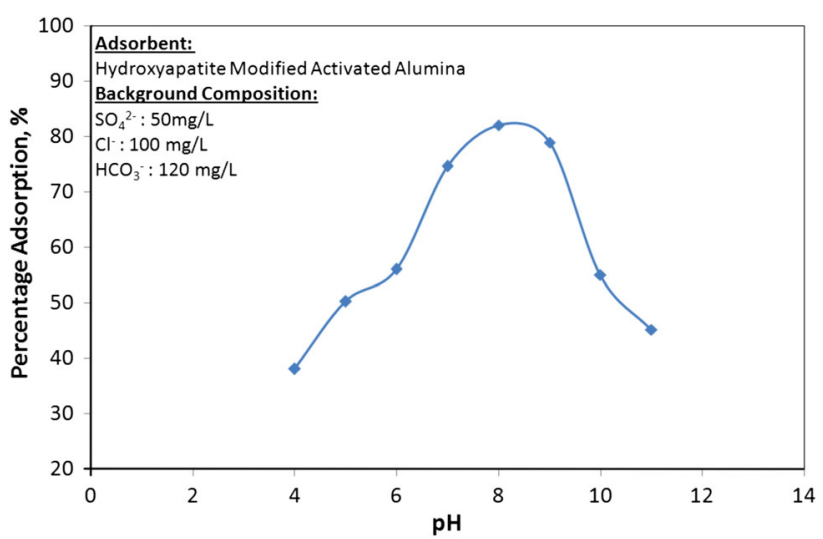

Fig. 5 Variation in fluoride adsorption capacity of hydroxyapatitemodified activated alumina at different $\mathrm{pH}$

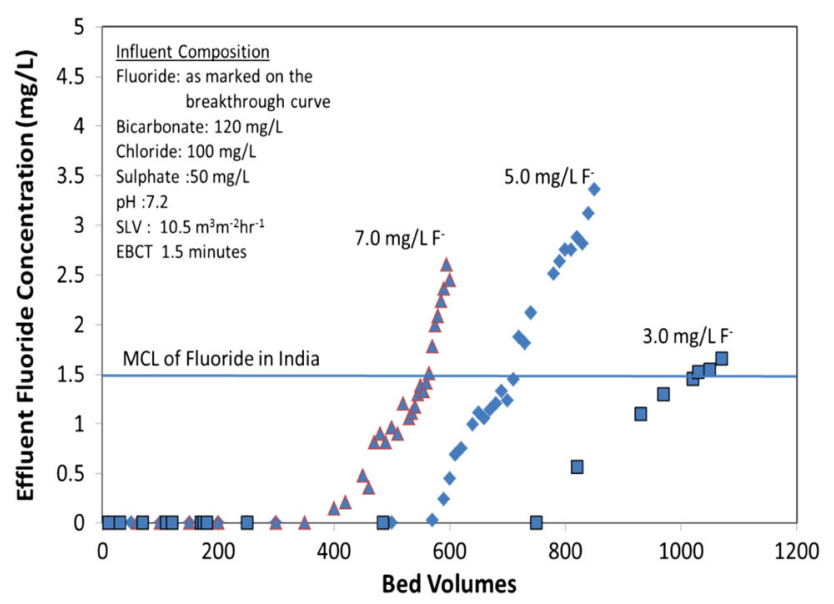

Fig. 6 Fluoride concentration histories in the effluent of a hydroxyapatite-modified activated alumina column run with solutions with different concentration of fluoride ( $S L V$ superficial liquid velocity, $E B C T$ empty bed contact time, $M C L$ maximum contaminant limit)

similar variation in its adsorption behavior with varying $\mathrm{pH}$, showing a maximum adsorption capacity at $\mathrm{pH}$ of 7 . The alkaline shift in the $\mathrm{pH}$ for maximum adsorption capacity for HMAA is probably attributable to the presence of hydroxyapatite, which is introduced as a dispersed phase within the pores of activated alumina. Decrease in the adsorption capacity at elevated $\mathrm{pH}$ as shown in Fig. 6 indicates that at higher $\mathrm{pH}$, there will be desorption of fluoride. This phenomenon was used for the development of process for the regeneration of the exhausted HMAA, which is described later in this article. 


\section{Column studies}

Fluoride-contaminated synthetic water with background of commonly occurring competing anions was passed through laboratory column (ACE Glass, USA) with internal diameter of $11 \mathrm{~mm}$ containing a bed of HMAA. A flow controller was used to control the flow rate of the solution through the column. Effluent water samples were collected at definite intervals of time at the bottom of the column and were analyzed for fluoride concentration. Figure 6 indicates the effluent histories of three separate column runs performed with synthetic solutions containing 3, 5 and $7 \mathrm{mg} / \mathrm{L}$ of fluoride. The breakthrough curves for all three solution concentrations showed sharp breakthrough patterns. The breakthrough of fluoride corresponding to influent concentration of 3,5 and $7 \mathrm{mg} / \mathrm{L}$ was observed to occur at 285, 350 and 400 bed volumes, respectively, and breakthrough of $1.5 \mathrm{mg} / \mathrm{L}$ fluoride occurred at 560, 680 and 110 bed volumes, respectively. Such a self-sharpening breakthrough pattern is characteristics of a favorable adsorption process, which was earlier demonstrated by the adsorption isotherm values of coefficients for which are shown in Table 1.

\section{Regeneration studies}

Once the effluent fluoride concentration rises above $1.5 \mathrm{mg} / \mathrm{L}$, the column run was discontinued. The exhausted HMAA bed after the column run was regenerated in situ using $1 \mathrm{~N} \mathrm{NaOH}$ solution having a $\mathrm{pH} 13$. It is evident from Fig. 5 that at high $\mathrm{pH}$, there is drastic reduction in the adsorption capacity. Figure 7 shows the effluent history of fluoride from the column during regeneration. The effluent history curve for the regeneration indicates that the regeneration was complete within six bed volumes. After the regeneration, the bed was rinsed with one bed volume of distilled water followed by passage of $0.5 \mathrm{M} \mathrm{HCl}$ until the effluent $\mathrm{pH}$ reaches a value of 7.0. The acid wash was necessary to protonate the adsorbents before proceeding with another adsorption run.

Mechanism of fluoride removal by hydroxyapatitemodified activated alumina (HMAA)

HMAA is essentially a hybrid adsorbent consisting of two different phases, AA and dispersed particles of

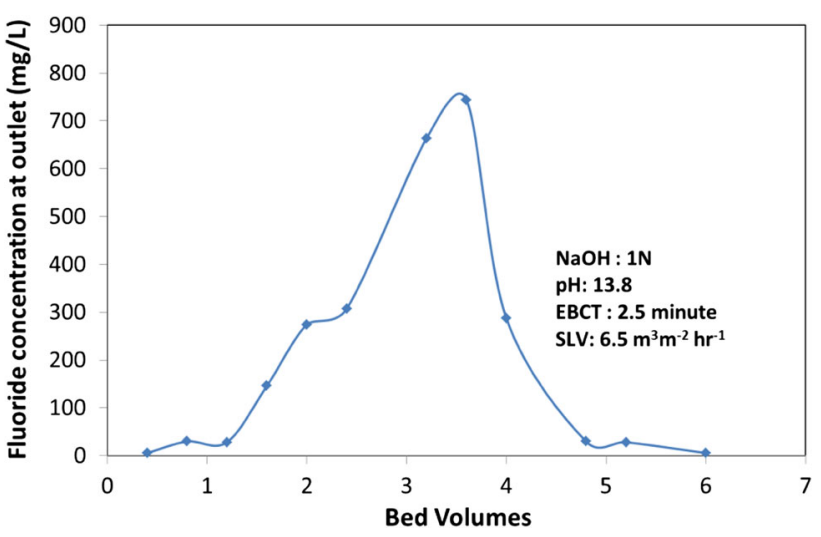

Fig. 7 Effluent fluoride concentration during regeneration of a column containing exhausted hydroxyapatite-modified activated alumina ( $S L V$ superficial liquid velocity, $E B C T$ empty bed contact time)

hydroxyapatite within the micropores of activated alumina. Hence, both the phases take part in the adsorption of fluoride. Adsorption on the aggregate adsorbent, HMAA, therefore can be explained by the following mechanism detailed in Eq. 5 through 10.

In AA phase following reactions take place (Ghorai and Pant 2004),

$\overline{\equiv \mathrm{AlOH}}+\mathrm{F}^{-} \Leftrightarrow \overline{\equiv \mathrm{AlF}}+\mathrm{OH}^{-}$
$\overline{\equiv \mathrm{AlOH}_{2}^{+}}+\mathrm{F}^{-} \Leftrightarrow \overline{\equiv \mathrm{AlF}}+\mathrm{H}_{2} \mathrm{O}$
$\overline{\equiv \mathrm{AlOH}}+2 \mathrm{~F}^{-} \Leftrightarrow \overline{\equiv \mathrm{AlF}_{2}^{-}}+\mathrm{OH}^{-}$

where, $\mathrm{Al}$ denotes aluminum at st denotes the solid phase. Reaction described in Eq. (11) takes place when there is high concentration of fluoride loading per unit weight of activated alumina.

The following reactions take place in the precipitated hydroxyapatite phase (MacDonald et al. 2011; Sarkar et al. 2012),

$$
\begin{aligned}
& \overline{\mathrm{Ca}_{5}\left(\mathrm{PO}_{4}\right)_{3}(\mathrm{OH})}+\mathrm{F}^{-} \Leftrightarrow \overline{\mathrm{Ca}_{5}\left(\mathrm{PO}_{4}\right)_{3}(\mathrm{OH}) \equiv \mathrm{F}^{-}} \\
& \overline{\mathrm{Ca}_{5}\left(\mathrm{PO}_{4}\right)_{3}(\mathrm{OH})}+\mathrm{F}^{-} \Leftrightarrow \overline{\mathrm{Ca}_{5}\left(\mathrm{PO}_{4}\right)_{3} \mathrm{~F}}+\mathrm{OH}^{-} \\
& \overline{\mathrm{Ca}_{5}\left(\mathrm{PO}_{4}\right)_{3} \mathrm{OH}_{2}^{+}}+\mathrm{F}^{-} \Leftrightarrow \overline{\mathrm{Ca}_{5}\left(\mathrm{PO}_{4}\right)_{3} \mathrm{OH}_{2}^{+} \mathrm{F}^{-}}
\end{aligned}
$$

Reactions in Eq. (6) and (10) take place at lower $\mathrm{pH}$, in abundance of $\mathrm{H}^{+}$ions.

During regeneration, high $\mathrm{pH}$ of the caustic solution within the micropores of the adsorbent caused the surface functional groups of AA to acquire net negative charge as 


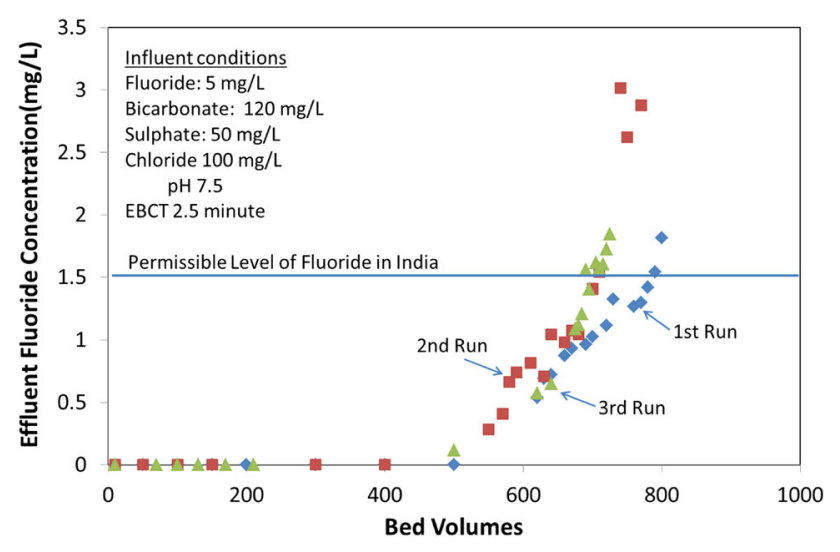

Fig. 8 Effluent fluoride histories of three consecutive runs of column containing hydroxyapatite-modified activated alumina subjected to influent solution with $5 \mathrm{mg} / \mathrm{L}$ fluoride (EBCT empty bed contact time)

the $\mathrm{pH}$ at the point of zero charge or $\mathrm{pH}_{\mathrm{PZC}}$ of $\mathrm{AA}$ is around 7.8 (Ramos et al. 2008) as it is evident from Figs. 5, 7. Presence of negatively charged fixed functional groups induces a Donnan exclusion potential, which would try to exclude the fluoride anions from inside the adsorbent phase (Sarkar et al. 2010) as shown in Eq. 11.

$\overline{\equiv \mathrm{AlF}}+\mathrm{OH}^{-} \Leftrightarrow \overline{\equiv \mathrm{AlO}^{-}}+\mathrm{F}^{-}+\mathrm{H}^{+}$

In the hydroxyapatite phase, the following reactions take place at elevated $\mathrm{pH}$ maintained during regeneration,

$\overline{\mathrm{Ca}_{5}\left(\mathrm{PO}_{4}\right)_{3} \mathrm{OH}_{2}^{+} \mathrm{F}^{-}}+\mathrm{OH}^{-} \rightarrow \overline{\mathrm{Ca}_{5}\left(\mathrm{PO}_{4}\right)_{3} \mathrm{OH} \equiv \mathrm{F}^{-}}+\mathrm{H}_{2} \mathrm{O}$

In abundance of $\mathrm{OH}^{-}$ions, the reactions 8,9 and 12 reverse in direction,

$\overline{\mathrm{Ca}_{5}\left(\mathrm{PO}_{4}\right)_{3}(\mathrm{OH}) \equiv \mathrm{F}^{-}} \rightarrow \overline{\mathrm{Ca}_{5}\left(\mathrm{PO}_{4}\right)_{3}(\mathrm{OH})}+\mathrm{F}^{-}$

$\overline{\mathrm{Ca}_{5}\left(\mathrm{PO}_{4}\right)_{3} \mathrm{~F}}+\mathrm{OH}^{-} \rightarrow \overline{\mathrm{Ca}_{5}\left(\mathrm{PO}_{4}\right)_{3}(\mathrm{OH})}+\mathrm{F}^{-}$

The reactions indicated in Eq. 11 through 14 indicate that at elevated $\mathrm{pH}$, the fluoride ions shall be essentially expelled from inside the adsorbent phase as observed in Figs. 5, 7. Following regeneration, a thorough rinse with water should completely expel the fluoride ions from the adsorbent columns. Before the media are put in operation again, there is a need to condition the regenerated media. The media were subjected to rinse with a solution of dilute $\mathrm{HCl}$ when the alumina surface gets protonated according to the following reactions, before it is reused again.

$\equiv \overline{\mathrm{AlO}^{-}}+\mathrm{H}^{+} \rightarrow \overline{\mathrm{AlOH}}$
$\equiv \mathrm{AlOH}+\mathrm{H}^{+} \rightarrow \overline{\mathrm{AlOH}_{2}^{+}}$

Consecutive cycles of operation

Consecutive column runs were performed with modified AA to validate its potential for reuse over multiple cycles of operation. The column runs were performed with the same influent solution each time, having a fluoride concentration of $5 \mathrm{mg} / \mathrm{L}$ with a background of commonly occurring anions. Once the adsorption column reaches breakthrough of $1.5 \mathrm{mg} / \mathrm{L}$ of fluoride, the column run was stopped and the exhausted media were regenerated in situ using the protocol mentioned above. The next run was performed with the regenerated HMAA. Figure 8 shows the fluoride breakthrough profiles from the column for three successive column runs, with all conditions remaining the same for all three runs. It may be observed that the column ran for almost the same amount of bed volumes over the three consecutive cycles of operation. This shows the potential of the adsorbent for reuse over multiple numbers of cycles without any appreciable loss in its removal capacity over repeated use.

\section{Conclusion}

Hydroxyapatite-modified activate alumina (HMAA) is a hybrid adsorbent prepared by dispersing nanoparticles of hydroxyapatite within the micropores of AA following chemical synthesis using inexpensive starting materials like calcium chloride and ammonium dihydrogen phosphate. The adsorbent showed higher capacity for removal of fluoride removal compared with its parent material, virgin-activated alumina, which is known as an effective adsorbent for fluoride ions. The introduction of hydroxyapatite precipitates within the pores of AA granules increased the number of surface active sites where adsorption of fluoride took place, effectively boosting the adsorption capacity of the hybrid adsorbent. Apart from a synergistic increase in the fluoride adsorption capacity, the hybrid adsorbent also showed excellent hydraulic characteristics, allowing the passage of water through a laboratory column, which was run 
under gravity. Also, the hybrid adsorbent was found to be amenable for regeneration using chemicals, which are low cost and easily available. The fluoride removal capacity was found to remain almost the same over three consecutive cycles of use. Thus, the hybrid adsorbent synthesized during the study may be considered to be a promising material for its practical use in the field for remediation of fluoride from contaminated drinking water. Further pilot-scale studies need to be performed in order to scale up the process for field use following a detailed engineering design.

Acknowledgments The second author, A.T., is supported by MHRD, GOI fellowship. Part of the work was supported by the Faculty Initiation Grant received from the corresponding authors Institute. The authors thankfully acknowledge the supports received from these grant and fellowships.

\section{References}

American Public Health Association (APHA) (2012) Standard methods for the examination of water and wastewater. American public health association, American water works association, water environment federation publication. 22nd edition. APHA, Washington D.C

Apparao BV, Meenakshi S, Karthikayan G (1998) Nalgonada technique of defluoridation of water Indian. J Environ Prot 10:292-298

Ayoob S, Gupta AK, Bhat VT (2008) A Conceptual overview on sustainable technologies for the defluoridation of drinking water. Crit Rev Environ Sci Tech 38:401-470

Azbar N, Turkman A (2000) Defluoridation in drinking waters. Water Sci Technol 42:403-407

Chernet T, Trafi Y, Valles V (2002) Mechanism of degradation of the quality of natural water in the lakes region of the Ethiopian rift valley. Water Res 35:2819-2832

Durmaz F, Kara H, Cengeloglu Y, Ersoz M (2005) Fluoride removal by Donnan dialysis with anion exchange membranes. Desalination 177:51-57

Farooqi A, Masuda H, Kusakabe M, Naseem M, Firdous N (2007) Distribution of highly arsenic and fluoride contaminated groundwater from east Punjab, Pakistan and the controlling role of anthropogenic pollutants in the natural hydrological cycle. Geochem J 41:213-234

Gao S, Cui J, Wei ZG (2009) Study on the fluoride adsorption of various apatite materials in aqueous solution. J Fluorine Chem 130:1035-1041

Ghorai S, Pant KK (2004) Investigations on the column performance of fluoride adsorption by activated alumina in a fixed bed. Chem Eng J 98:165-173
Ghorai S, Pant KK (2005) Equilibrium, kinetics and breakthrough studies for adsorption of fluoride on activated alumina. Sep Purif Technol 42:265-271

Hichour M, Persin F, Sandeaux J, Gavach C (2000) Fluoride removal from waters by Donnan dialysis. Sep Purif Technol 18:1-11

Hou D, Wang J, Zhao C, Wang B, Luan Z, Sun X (2010) Fluoride removal from brackish groundwater by direct contact membrane distillation. J Environ Sci 22:1860-1867

Hu K, Dickson JM (2006) Nanofiltration membrane performance on fluoride removal from water. J Membr Sci 279:529-538

Kabay N, Arar O, Samatya S, Yüksel U, Yüksel M (2008) Separation of fluoride from aqueous solution by electrodialysis: effect of process parameters and other ionic species. J Hazard Mater 153:107-113

MacDonald LH, Pathak G, Singer B, Jaffe PR (2011) An integrated approach to address endemic fluorosis in Jharkhand, India. J Water Res Protection 3:457-472

Malaisamy R, Talla-Nwafo A, Jones KL (2011) Polyelectrolyte modification of nanofiltration membrane for selective removal of monovalent anions. Sep Purif Technol 77:367-374

Meenakshi S, Maheshwari RC (2006) Fluoride in drinking water and its removal. J Hazard Mater 137:456-463

Mohapatra M, Anand S, Mishra BK, Giles DE, Singh P (2009) Review of fluoride removal from drinking water. J Environ Manage 91:67-77

Ndiaye PI, Moulin P, Dominguez L, Millet JC, Charbit F (2005) Removal of fluoride from electronic industrial effluent by RO membrane separation. Desalination 173:25-32

Ramos RL, Castillo NAM, Azuara AJ, Barron JM, Rodriguez LEL, Rosales JMM, Pina AA (2008) Fluoride removal from water solution by adsorption on activated alumina prepared from pseudo-boehmite. J Environ Eng Manage 18:301-309

Rao NV, Mohan R, Bhaskaran CS (1998) Studies on defluoridation of water. J Fluorine Chem 41:17-24

Reardon EL, Wang Y (2000) A limestone reactor for fluoride removal from wastewaters. Environ Sci Technol 34:3247-3253

Rehman I, Bonfield W (1997) Characterization of hydroxyapatite and carbonated apatite by photo acoustic FTIR spectroscopy. J Mater Sci Mater Med 8:1-4

Richards LA, Vuachère M, Schäfer AI (2010) Impact of pH on the removal of fluoride, nitrate and boron by nanofiltration/reverse osmosis. Desalination 261:331-337

Saha S (1993) Treatment of aqueous effluent for fluoride removal. Water Res 27:1347-1350

Sahli MA, Annouar A, Tahaikt S, Mountadar M, Soufiane A, Elmidaoui A (2007) Fluoride removal for underground brackish water by adsorption on the natural chitosan and by electrodialysis. Desalination 212:37-45

Sarkar S, Prakash P, SenGupta AK (2010) The donnan membrane principle: opportunities for sustainable engineered processes and materials. Environ Sci Technol 44:1161-1166

Sarkar S, Guibal E, Quignard F, SenGupta AK (2012) Polymersupported metals and metal oxide nanoparticles: synthesis, characterization, and applications. J Nanoparticle Res 14:715

Singh G, Kumar B, Sen PK, Majumdar J (1999) Removal of fluoride from spent pot liner leachate using ion exchange. Water Environ Res 71:36-42 
Stuart BH (2004) Infrared spectroscopy: fundamentals and applications. Wiley, Australia, pp 72-152

Sundaram CS, Viswanathan N, Meenakshi S (2008) Defluoridation chemistry of synthetic hydroxyapatite at nano scale: equilibrium and kinetic studies. J Hazard Mater 155:206-215

Sundaram CS, Viswanathan N, Meenakshi S (2009) Fluoride sorption by nanohydroxyapatite/chitin composite. J Hazard Mater 172:147-151
Wang WY, Li RB, Tan JA, Luo KL, Yang LS, Li HR, Li YH (2002) Adsorption and leaching of fluoride in soils of China. Fluoride 35:122-129

World Health Organization (2008) Guidelines for drinking-water quality: third edition, Incorporating the first and second addenda. 3rd edition, Geneva 